\title{
BEM+Born series modeling schemes for wave propagation and their convergence analysis*
}

\author{
Gengxin $\mathrm{Yu}^{*}$ Liyun $\mathrm{Fu}$ and Xizhu Guan \\ Institute of Geology and Geophysics, Chinese Academy of Sciences, Beijing 100029, China
}

\begin{abstract}
This paper formulates two different boundary-element+Born series schemes for wave propagation simulation in multilayered media by incorporating a Born series and boundary integral equations. The first scheme directly decomposes the resulting boundary integral equation matrix into the self-interaction operators associated with each boundary itself and the extrapolation operators expressing cross-interactions between different boundaries in a subregion. For the second scheme, the matrix dimension is firstly reduced to a half by the elimination of the traction field in the equations. The resulting new matrix can also be split into the self-interaction matrices associated each subregion itself and the extrapolation matrices interpreting cross-interactions between different subregions in a whole model. Both the numerical schemes avoid the inversion of the relatively much larger boundary integral equation matrix of a full-waveform BE method and hence save computing time and memory greatly. The two schemes are validated by calculating synthetic seismograms for a homogeneous layered model, compared with the full-waveform BE numerical solution. Numerical experiments indicate that both the BEM+Born series modeling schemes are valid and effective. The tests also confirm that the second modeling scheme has a faster convergence in comparison with the first one.
\end{abstract}

Key words: boundary-element method; Born series; self-interaction operator; extrapolation operator CLC number: $\mathrm{P} 315.3^{+} 1 \quad$ Document code: A

\section{Introduction}

In general, complex geological structures consist of irregular subregions with piecewise constant properties. Irregular interfaces separate different geological formations. These strong-contrast impedance boundaries control the principal characteristics of wave propagation and pose a special requirement for seismic modeling schemes to simulate subtle reflection/transmission effects across interfaces. Boundary-element (BE) methods enjoy a distinct characteristic of the explicit use of the boundary conditions, which provides sufficient accuracy in the simulation of reflection/transmission across irregular interfaces (Fu, 2002). Therefore, BE methods have been applied to 2D and 3D seismic models in exploration geophysics (e.g., Bakamjian, 1992; Bouchon, 1993; Chen and Zhou, 1994; Fu and Mu, 1994; Dong et al, 1995; Fu, 1996; Liu et al, 2008a, b; Yu et al, 2009). However the BE methods have computational limitations for large or complex models and are often limited

\footnotetext{
* Received 9 November 2009; accepted in revised form 14 January 2010; published 10 April 2010.

+Corresponding author. e-mail: y_g_xin@126.com

(C) The Seismological Society of China and Springer-Verlag Berlin Heidelberg 2010
}

to cases of low frequencies. At higher frequencies, the computation becomes extremely prohibitive because of the very large size of the resulting matrices to be inverted. An efficient alternative is to incorporate Born series approximation into the BE methods to overcome the computational barrier. This paper aims to formulating BEM+Born series modeling schemes for irregular multilayered media.

Several flexible approximation solutions have been developed from the full-waveform BE modeling methods, with a great saving of computing time and memory. In a series of articles (Schuster, 1985a, b; Schuster and Smith, 1985), an elegant hybrid method in solving multibody scattering problems is proposed by incorporating a Born series and boundary integral equations. The hybrid BEM+Born series method perturbs the boundary integral equation matrix into two parts, one being easily inverted and physically associated with the self-interaction operators of the scatterers that are uncoupled from each other, and the other often being not inverted at all and corresponding to the extrapolation operators across free space. The method avoids the inversion of the relatively much larger boundary integral equation matrix of a pure BE method, and hence improves the computational effi- 
ciency greatly (Schuster, 1985a). Based on the Schuster and Smith (1985) strategy, this paper formulates two different hybrid BEM+Born series modeling schemes for wave propagation simulation in multilayered media. The first scheme directly decomposes the resulting boundary integral equation matrix into the self-interaction operators associated with each boundary itself and the extrapolation operators expressing cross-interactions between different boundaries in a subregion. For the second scheme, the matrix dimension is first reduced to a half by the elimination of the traction field in the equations. The resulting new matrix can also be split into the self-interaction matrices associated each subregion itself and the extrapolation matrices interpreting cross-interactions between different subregions in a whole model. The two schemes are validated by calculating synthetic seismograms for a homogeneous layered model, compared with the full-waveform BE numerical solution. Furthermore, the convergence of the two modeling schemes is discussed in this paper.

\section{Method}

\subsection{Boundary integral eqution for multilayered media}

Wave propagation in a boundary structure can be described by a boundary integral equation. The problem to be studied is illustrated in Figure 1. In this model, there are $n$ homogeneous layers over a half-space, among which the $i$ th layer $\Omega_{i}$ is bounded by the top interface $\Gamma_{i-1}$ and the bottom interface $\Gamma_{i}$. The side boundaries at two edges of $\Omega_{i}$ are assumed to extend to infinity. The uppermost interface $\Gamma_{0}$ is a free surface, and an arbitrary source is embedded in the $i$ th layer. For simplicity, the present study is restricted to the $2 \mathrm{D}$ acoustic problem. The solution domain of the problem is defined as $\bar{\Omega}_{i} \in \Gamma_{i-1}+\Gamma_{i}$. Seismic response $u(\boldsymbol{r})$ for steady state scalar wave propagation in the $i$ th layer satisfies the following scalar equation

$$
\nabla^{2} u(\boldsymbol{r})+\left[k_{0}^{(i)}\right]^{2} u(\boldsymbol{r})=-s^{(i)}(\boldsymbol{r}, \omega),
$$

where $k_{0}^{(i)}=\omega / v_{0}^{(i)}$ is the background wavenumber, $v_{0}^{(i)}$ is the velocity of the $i$ th layer, $\omega$ is the frequency, $s(\boldsymbol{r}, \omega)$ is the source term and $\nabla^{2}$ is the Laplacian operator. The seismic response $u(r)$ also satisfies the following boundary conditions. (1) The traction-free condition on the free surface: $\partial u / \partial n=0$ at $r \in \Gamma_{0}$; (2) The continuities of displacement and traction at the interface $\Gamma_{i}$,

$$
\left\{\begin{array}{c}
u_{-}^{(i)}(\boldsymbol{r})=u_{+}^{(i)}(\boldsymbol{r}) \\
\frac{\partial u_{-}^{(i)}(\boldsymbol{r})}{\partial n}=\frac{\partial u_{+}^{(i)}(\boldsymbol{r})}{\partial n}, \quad \boldsymbol{r} \in \Gamma_{i}
\end{array}\right.
$$

where ' - ' denotes the top side of $\Gamma_{i}$ toward $\Omega_{i}$ and ' + ' denotes the underside of $\Gamma_{i}$ toward $\Omega_{i+1}$.

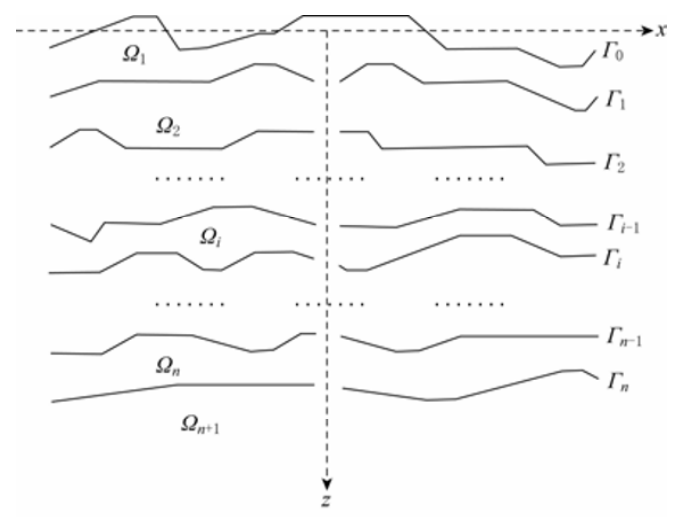

Figure 1 Configuration of the problem considered.

(3) The radiation boundary condition imposed on the far-field behavior at infinity.

$$
\left\{\begin{array}{l}
\lim _{|r| \rightarrow \infty} u(\boldsymbol{r})=0 \\
\lim _{|r| \rightarrow \infty} \frac{\partial u(\boldsymbol{r})}{\partial r}=0
\end{array} .\right.
$$

To explicitly use the boundary condition defined by equation (2) in the solution of the problem, we need to transform equation (1) into an integral equation in terms of boundary integrals of the solution domain. Supposing the source point is located at $\boldsymbol{r}_{0}$, the source term can be expressed as $s(\boldsymbol{r}, \omega)=S(\omega) \delta\left(\boldsymbol{r}-\boldsymbol{r}_{0}\right)$, where $S(\omega)$ is the source spectrum and $\delta\left(\boldsymbol{r}-\boldsymbol{r}_{0}\right)$ is the delta function. According to integral equation theory (Pao and Varatharajulu, 1976), and considering the "boundary naturalization" of the integral equations (that is, a limit analysis when the "observation point" $\boldsymbol{r}=(x, z)$ approaches the boundary $\Gamma_{i}$ and tends to coincide with the "scattering point" $\boldsymbol{r}^{\prime}=\left(x^{\prime}, z^{\prime}\right)$ when $\left.\boldsymbol{r}^{\prime} \in \Gamma_{i}\right)$, we obtain the following boundary integral equation

$$
\begin{aligned}
& \int_{\Gamma_{i-1}}\left[G^{(i)}\left(\boldsymbol{r}, \boldsymbol{r}^{\prime}\right) t^{(i-1)}\left(\boldsymbol{r}^{\prime}\right)-u^{(i-1)}\left(\boldsymbol{r}^{\prime}\right) \frac{\partial G^{(i)}\left(\boldsymbol{r}, \boldsymbol{r}^{\prime}\right)}{\partial n}\right] \mathrm{d} \boldsymbol{r}^{\prime} \\
& +\int_{\Gamma_{i}}\left[G^{(i)}\left(\boldsymbol{r}, \boldsymbol{r}^{\prime}\right) t^{(i)}\left(\boldsymbol{r}^{\prime}\right)-u^{(i)}\left(\boldsymbol{r}^{\prime}\right) \frac{\partial G^{(i)}\left(\boldsymbol{r}, \boldsymbol{r}^{\prime}\right)}{\partial n}\right] \mathrm{d} \boldsymbol{r}^{\prime} \\
& +S(\omega) G^{(i)}\left(\boldsymbol{r}, \boldsymbol{r}_{0}\right)= \begin{cases}C^{(i-1)}(\boldsymbol{r}) u^{(i-1)}(\boldsymbol{r}) & \boldsymbol{r} \in \Gamma_{i-1} \\
C^{(i)}(\boldsymbol{r}) u^{(i)}(\boldsymbol{r}) & \boldsymbol{r} \in \Gamma_{i}\end{cases}
\end{aligned}
$$


for all $\boldsymbol{r}^{\prime} \in \bar{\Omega}_{i}$, where $u^{(i)}(\boldsymbol{r})$ is the displacement on $\Gamma_{i}$, $t^{(i)}(\boldsymbol{r})=\partial u^{(i)}(\boldsymbol{r}) / \partial n$ is the normal gradient of the displacement on $\Gamma_{i}$, and the coefficients $C(\boldsymbol{r})$ generally depend on the local geometry at $\boldsymbol{r}$. The causal Green's function is defined everywhere in the free space, relating an observation point $\boldsymbol{r}$ to a scattering point $\boldsymbol{r}^{\prime}$. It satisfies the homogeneous Helmholtz equation in the reference medium

$$
\begin{gathered}
\nabla^{2} G^{(i)}\left(\boldsymbol{r}, \boldsymbol{r}^{\prime}\right)+\left[k_{0}^{(i)}\right]^{2} G^{(i)}\left(\boldsymbol{r}, \boldsymbol{r}^{\prime}\right)=-\delta\left(\boldsymbol{r}-\boldsymbol{r}^{\prime}\right), \\
\boldsymbol{r}, \boldsymbol{r}^{\prime} \in \bar{\Omega}_{i} .
\end{gathered}
$$

For 2D problems, the Green's function is given by (Abramowitz and Stegun, 1968)

$$
G^{(i)}\left(\boldsymbol{r}, \boldsymbol{r}^{\prime}\right)=\frac{i}{4} H_{0}^{(1)}\left(k_{0}^{(i)}\left|\boldsymbol{r}^{\prime}-\boldsymbol{r}\right|\right),
$$

where $i=\sqrt{-1}$ and $H_{0}^{(1)}$ denotes the Hankel function of the first kind and of zeroth order. The integral equation (4) naturally satisfies Sommerfeld non-reflecting and decay boundary conditions defined by equation (3).

\subsection{Numerical discretization of boundary integral} equation

In the frequency-space domain BE method, each boundary $\Gamma_{i}$ is divided into $N_{E}^{(i)}$ linear elements denoted by $\Gamma_{e}\left(e=1,2, \ldots, N_{E}^{(i)}\right)$. The total node number of $\bar{\Omega}_{i}$ is $N_{P}^{(i)}$. By using linear interpolation shape functions $N(\xi)$ in an element $\Gamma_{e}$ between the node $I_{1}$ and $I_{2}$, we obtain

$$
u^{(i)}(\xi)=\sum_{l=I_{1}}^{I_{2}} u^{(i)}\left(\boldsymbol{r}_{l}\right) N_{l}(\xi),
$$

where $\xi$ denotes the local coordinate of an element, and $l$ denotes the local node index of an element. The integrals in equation (4) can be computed over each element respectively as

$$
\mid \begin{array}{rrr}
\boldsymbol{H}_{11}^{1}-\boldsymbol{G}_{11}^{1} & & \\
\boldsymbol{H}_{11}^{2}-\boldsymbol{G}_{11}^{2} & \boldsymbol{H}_{12}^{2} & -\boldsymbol{G}_{12}^{2} \\
\boldsymbol{H}_{21}^{2}-\boldsymbol{G}_{21}^{2} & \boldsymbol{H}_{22}^{2} & -\boldsymbol{G}_{22}^{2} \\
& \ddots & \ddots \\
& & \boldsymbol{H}^{\prime} \\
& & \\
& & \\
& & \\
& &
\end{array}
$$

$$
\begin{gathered}
h_{j k}^{(i, 1)}=\sum_{e=1}^{N_{k}^{(i-1)}} \sum_{l=I_{1}}^{I_{2}}\left[\int_{\Gamma_{e}} \frac{\partial}{\partial n} G^{(i)}\left(\boldsymbol{r}_{j}, \boldsymbol{r}^{\prime}(\xi)\right) N_{l}(\xi) \mathrm{d} \boldsymbol{r}^{\prime}(\xi)\right] \delta_{l k}+ \\
C^{(i-1)}\left(\boldsymbol{r}_{j}\right) \delta_{j k}, \\
g_{j k}^{(i, 1)}=\sum_{e=1}^{N_{E}^{(i-1)}} \sum_{l=I_{1}}^{I_{2}}\left[\int_{\Gamma_{e}} G^{(i)}\left(\boldsymbol{r}_{j}, \boldsymbol{r}^{\prime}(\xi)\right) N_{l}(\xi) \mathrm{d} \boldsymbol{r}^{\prime}(\xi)\right] \delta_{l k},
\end{gathered}
$$

$$
\begin{gathered}
h_{j k}^{(i, 2)}=\sum_{e=1}^{N_{k}^{(i)}} \sum_{l=I_{1}}^{I_{2}}\left[\int_{\Gamma_{e}} \frac{\partial}{\partial n} G^{(i)}\left(\boldsymbol{r}_{j}, \boldsymbol{r}^{\prime}(\xi)\right) N_{l}(\xi) \mathrm{d} \boldsymbol{r}^{\prime}(\xi)\right] \delta_{l k}+ \\
C^{(i)}\left(\boldsymbol{r}_{j}\right) \delta_{j k},
\end{gathered}
$$

$$
g_{j k}^{(i, 2)}=\sum_{e=1}^{N_{E}^{(i)}} \sum_{l=I_{1}}^{I_{2}}\left[\int_{\Gamma_{e}} G^{(i)}\left(\boldsymbol{r}_{j}, \boldsymbol{r}^{\prime}(\xi)\right) N_{l}(\xi) \mathrm{d} \boldsymbol{r}^{\prime}(\xi)\right] \delta_{l k},
$$

where $\delta_{l k}$ and $\delta_{j k}$ are the Kronecker delta functions. The Gaussian integration algorithm is used to numerically evaluate these integrals. Equation (4) can be written in an operator form

$$
\begin{gathered}
\boldsymbol{H}^{(i, 1)} \boldsymbol{u}^{(i-1)}\left(\boldsymbol{r}_{j}\right)-\boldsymbol{G}^{(i, 1)} \boldsymbol{t}^{(i-1)}\left(\boldsymbol{r}_{j}\right)+\boldsymbol{H}^{(i, 2)} \boldsymbol{u}^{(i)}\left(\boldsymbol{r}_{j}\right)- \\
\boldsymbol{G}^{(i, 2)} \boldsymbol{t}^{(i)}\left(\boldsymbol{r}_{j}\right)=\boldsymbol{f}\left(\boldsymbol{r}_{j}\right), \quad j=1,2, \cdots, N_{P}^{(i)},
\end{gathered}
$$

where $\boldsymbol{f}$ is the incident field, $\boldsymbol{H}^{(i, l)}$ and $\boldsymbol{G}^{(i, l)}$ are the top-boundary coefficient matrices composed of $h_{j k}^{(i, 1)}$ and $g_{j k}^{(i, 1)}$, and $\boldsymbol{H}^{(i, 2)}$ and $\boldsymbol{G}^{(i, 2)}$ are the bottom-boundary coefficient matrices consisting of $h_{j k}^{(i, 2)}$ and $g_{j k}^{(i, 2)}$.

Considering a multilayered model with the source located in the shallowest layer as the case in seismic exploration, one will obtain a series of matrix equations by applying equation (12) to all the subregions along from $\Omega_{1}$ to $\Omega_{n+1}$. Application of the boundary conditions such as the continuities of displacement and stress across all the interfaces will compact these matrix equations into a global matrix equation system (13)

$$
\begin{aligned}
& \boldsymbol{H}_{j j}^{i}-\boldsymbol{G}_{j j}^{i} \boldsymbol{H}_{j k}^{i}-\boldsymbol{G}_{j k}^{i} \\
& \boldsymbol{H}_{k j}^{i}-\boldsymbol{G}_{k j}^{i} \boldsymbol{H}_{k k}^{i}-\boldsymbol{G}_{k k}^{i}
\end{aligned}
$$

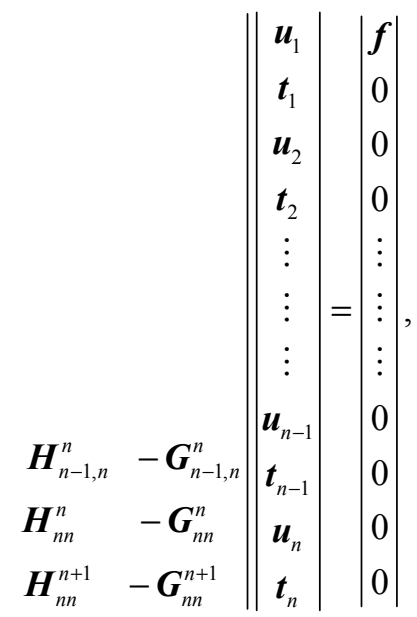


where $\boldsymbol{H}_{j k}^{i}$ and $\boldsymbol{G}_{j k}^{i}$ denote contour integrations over the interface $\Gamma_{k}$ while the observation point resides on boundary $\Gamma_{j}$ in the $i$ th subregion. After solving this matrix equation for $\boldsymbol{u}_{1}$ and $\boldsymbol{t}_{1}$, one will be able to calculate the value of the observed field anywhere inside $\Omega_{1}$ by direct substitution of equation (4).

\subsection{The first BEM+Born series modeling scheme}

The boundary integral equation (12) can be written as

\section{$\boldsymbol{H u}-\mathrm{Gt}=\boldsymbol{f}$.}

We define the boundary coefficient matrices $\boldsymbol{A}=[\boldsymbol{H},-\boldsymbol{G}]$, and the unknown boundary displacement-traction vector $\boldsymbol{m}=[\boldsymbol{u}, \boldsymbol{t}]$, then equation (14) can be expressed as

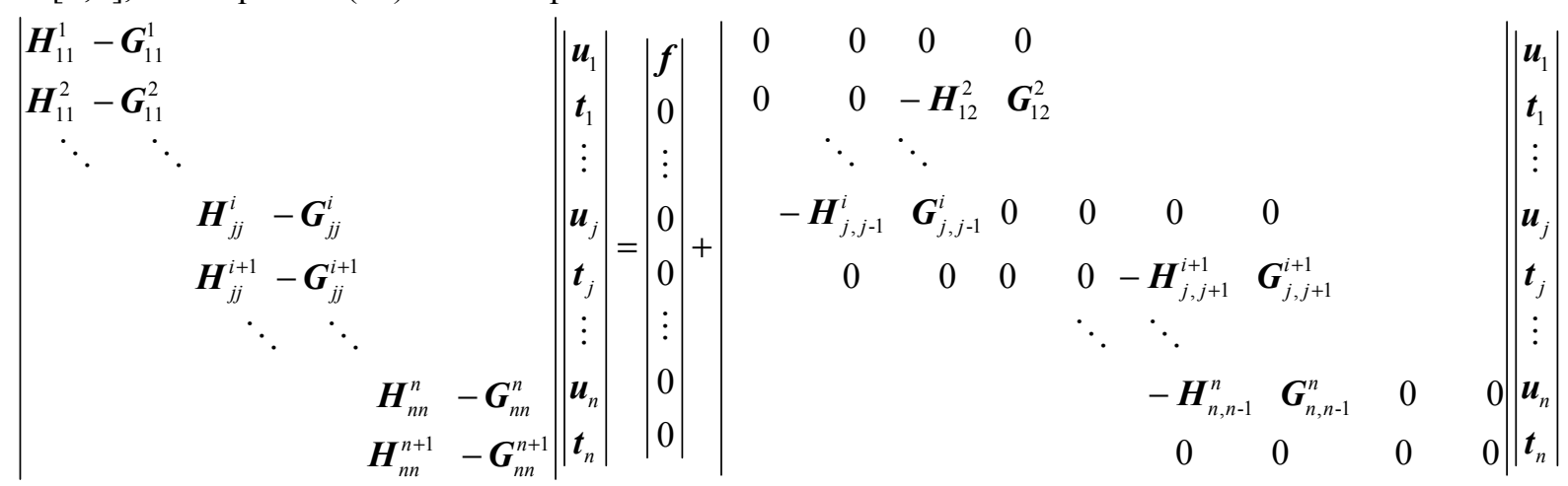

For the multilayered model, equation (13) can be rewritten as

According to the first BEM+Born series scheme, the matrix $\boldsymbol{A}$ can be split into self-interaction type matrices $\boldsymbol{S}$, and block-banded extrapolation matrices $\boldsymbol{E}=\boldsymbol{S}-\boldsymbol{A}$, then equation (15) becomes

$$
\boldsymbol{S} \boldsymbol{m}=\boldsymbol{f}+\boldsymbol{E} \boldsymbol{m} .
$$

Equation (16) can be approximated by the Born series (Schuster, 1985a)

$$
\boldsymbol{m}=\sum_{i=0}^{\infty}\left(\boldsymbol{S}^{-1} \boldsymbol{E}\right)^{i} \boldsymbol{S}^{-1} \boldsymbol{f}
$$

For $j=k$, we define $\boldsymbol{S}_{j}=\left[\begin{array}{cc}\boldsymbol{H}_{j j}^{i} & -\boldsymbol{G}_{i j}^{i} \\ \boldsymbol{H}_{j j}^{i+1} & -\boldsymbol{G}_{j j}^{i+1}\end{array}\right]$, where $\boldsymbol{S}_{j}$ is related to the interaction of the $j$ th boundary with itself; for $j \neq k$, we define $\boldsymbol{E}_{j k}=\left[\begin{array}{cc}-\boldsymbol{H}_{j k}^{i} & \boldsymbol{G}_{j k}^{i} \\ \boldsymbol{0} & \boldsymbol{0}\end{array}\right]$ or $\boldsymbol{E}_{j k}=\left[\begin{array}{cc}0 & 0 \\ -\boldsymbol{H}_{j k}^{i+1} & \boldsymbol{G}_{j k}^{i+i}\end{array}\right]$, where $\boldsymbol{E}_{j k}$ is related to the cross-interaction of the $j$ th boundary with the $k$ th boundary. Let $\boldsymbol{m}=\left[\boldsymbol{u}_{i}, \boldsymbol{t}_{i}\right]$, then equation (18) can be transformed into

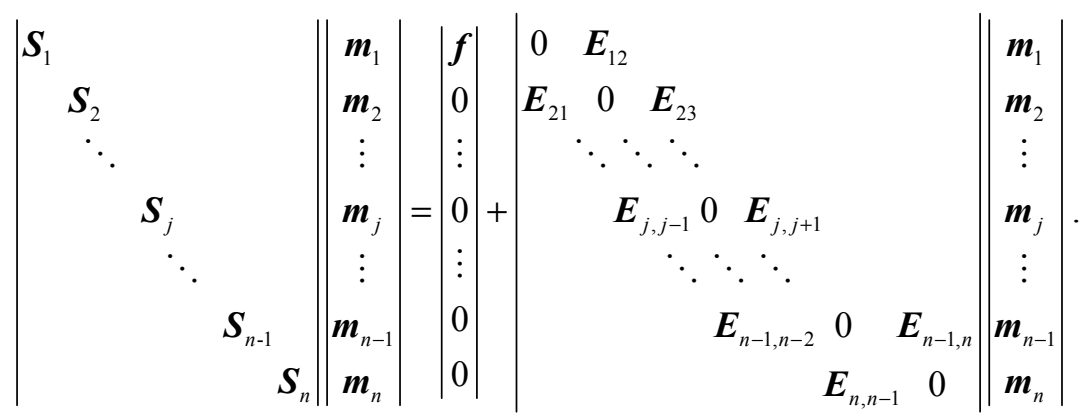

Using the Born series approximation, then we get

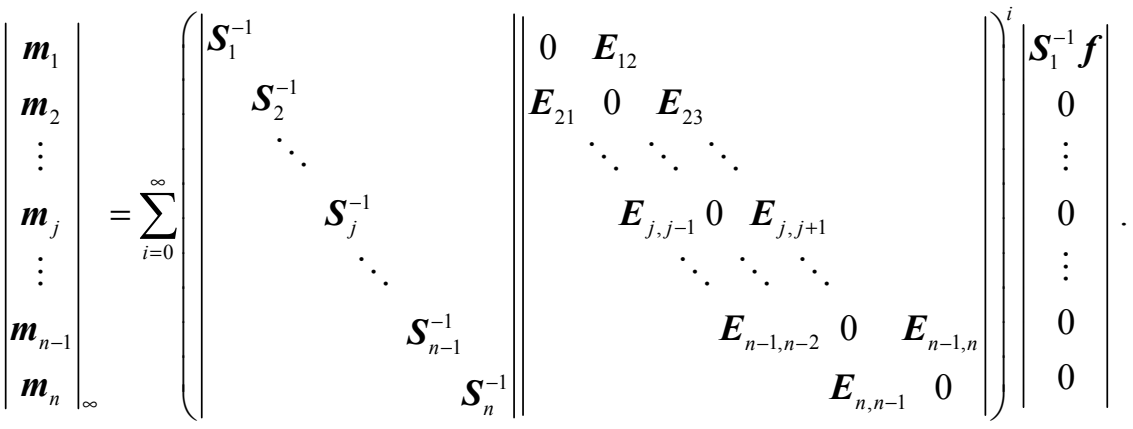


Taking the model in Figure 2 for an example, the Born series form can be expressed as

$$
\begin{aligned}
& \left|\begin{array}{l}
\boldsymbol{m}_{1} \\
\boldsymbol{m}_{2} \\
\boldsymbol{m}_{3}
\end{array}\right|_{\infty}=\sum_{i=0}^{\infty}\left(\left|\begin{array}{lll}
\boldsymbol{S}_{1}^{-1} & & \\
& \boldsymbol{S}_{2}^{-1} & \\
& & \boldsymbol{S}_{3}^{-1}
\end{array}\right|\left|\begin{array}{ccc}
0 & \boldsymbol{E}_{12} & 0 \\
\boldsymbol{E}_{21} & 0 & \boldsymbol{E}_{23} \\
0 & \boldsymbol{E}_{32} & 0
\end{array}\right|\right)^{i}\left|\begin{array}{c}
\boldsymbol{S}_{1}^{-1} \boldsymbol{f} \\
0 \\
0
\end{array}\right| .
\end{aligned}
$$

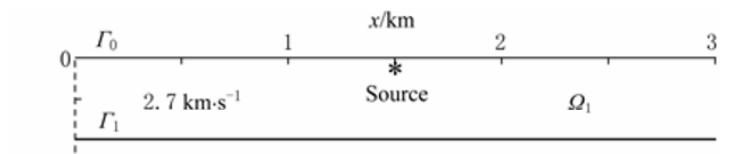

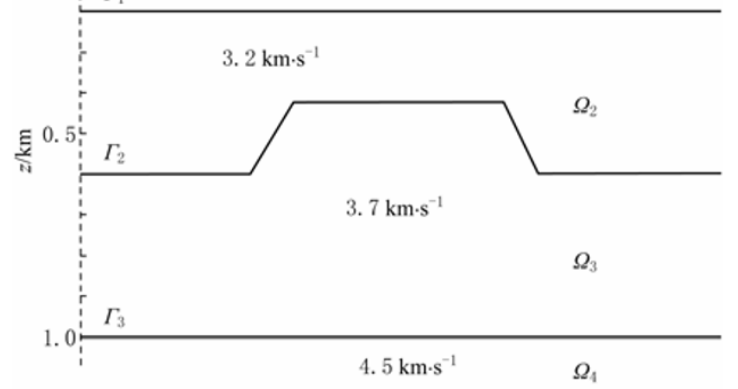

Figure 2 A homogeneous multilayered model.

\subsection{The second BEM+Born series modeling scheme}

For the second BEM+Born series scheme, the first step is to reduce the matrix dimension by the elimination of the traction field $t$, obtaining an integral equation that contains the displacement field $\boldsymbol{u}$ only. We can express the new integral equation in operator form

$$
\boldsymbol{A}^{\prime} \boldsymbol{u}=\boldsymbol{f}
$$

The matrix $\boldsymbol{A}^{\prime}$ can also be split into self-interaction type matrices $S^{\prime}$, and block-banded extrapolation matrices $\boldsymbol{E}^{\prime}=\boldsymbol{S}^{\prime}-\boldsymbol{A}^{\prime}$, then equation (22) becomes

$$
\boldsymbol{S}^{\prime} \boldsymbol{u}=\boldsymbol{f}+\boldsymbol{E}^{\prime} \boldsymbol{u}
$$

Similarly, equation (23) can be approximated by the Born series

$$
\boldsymbol{u}=\sum_{i=0}^{\infty}\left(\boldsymbol{S}^{\prime-1} \boldsymbol{E}^{\prime-1}\right)^{i} \boldsymbol{S}^{\prime-1} \boldsymbol{f}
$$

For the model depicted in Figure 1, the boundary integral equation for the observation points over boundary $\Gamma_{n}$ in $\Omega_{n+1}$ can be written as

$$
\boldsymbol{H}_{n n}^{n+1} \boldsymbol{u}_{n}-\boldsymbol{G}_{n n}^{n+1} \boldsymbol{t}_{n}=0 .
$$

The traction vector $\boldsymbol{t}_{n}$ in equation (25) can be expressed as

$$
\boldsymbol{t}_{n}=\left(\boldsymbol{G}_{n n}^{n+1}\right)^{-1} \boldsymbol{H}_{n n}^{n+1} \boldsymbol{u}_{n} .
$$

Letting $\boldsymbol{L}=(\boldsymbol{G})^{-1} \boldsymbol{H}$, then we have

$$
\boldsymbol{t}_{n}=\boldsymbol{L}_{n n} \boldsymbol{u}_{n} .
$$

Similar to equation (25), the boundary integral equation for the observation points over boundary $\Gamma_{n-1}$ in $\Omega_{n}$ can be written as

$$
\boldsymbol{H}_{n-1, n-1}^{n} \boldsymbol{u}_{n-1}-\boldsymbol{G}_{n-1, n-1}^{n} \boldsymbol{t}_{n-1}+\boldsymbol{H}_{n-1, n}^{n} \boldsymbol{u}_{n}-\boldsymbol{G}_{n-1, n}^{n} \boldsymbol{t}_{n}=0
$$

Substituting equation (27) into (28), then we have

$$
\boldsymbol{t}_{n-1}=\boldsymbol{L}_{n-1, n-1} \boldsymbol{u}_{n-1}+\left(\boldsymbol{G}_{n-1, n-1}^{n}\right)^{-1} \boldsymbol{M}_{n-1, n} \boldsymbol{u}_{n},
$$

where $\boldsymbol{M}=\boldsymbol{H}-\boldsymbol{G} \boldsymbol{L}$.

Doing as this, then we can get

$$
\begin{gathered}
\boldsymbol{t}_{j}=\boldsymbol{L}_{j j} \boldsymbol{u}_{j}+\left(\boldsymbol{G}_{j j}^{i}\right)^{-1} . \\
{\left[\boldsymbol{M}_{j, j+1} \boldsymbol{u}_{j+1}+\boldsymbol{N}_{j, j+2} \boldsymbol{u}_{j+2}+\cdots+\boldsymbol{P}_{j, n} \boldsymbol{u}_{n}\right] .}
\end{gathered}
$$

where $\boldsymbol{N}=\left(\boldsymbol{G} \boldsymbol{G}^{-1}\right) \boldsymbol{M}$ and $\boldsymbol{P}=\overbrace{\left(\boldsymbol{G} \boldsymbol{G}^{-1}\right) \cdots\left(\boldsymbol{G} \boldsymbol{G}^{-1}\right)}^{n-j-1} \boldsymbol{M}$.

A new matrix equation that contains the displacement field $\boldsymbol{u}$ only can be obtained by eliminating all the traction term in equation (13)

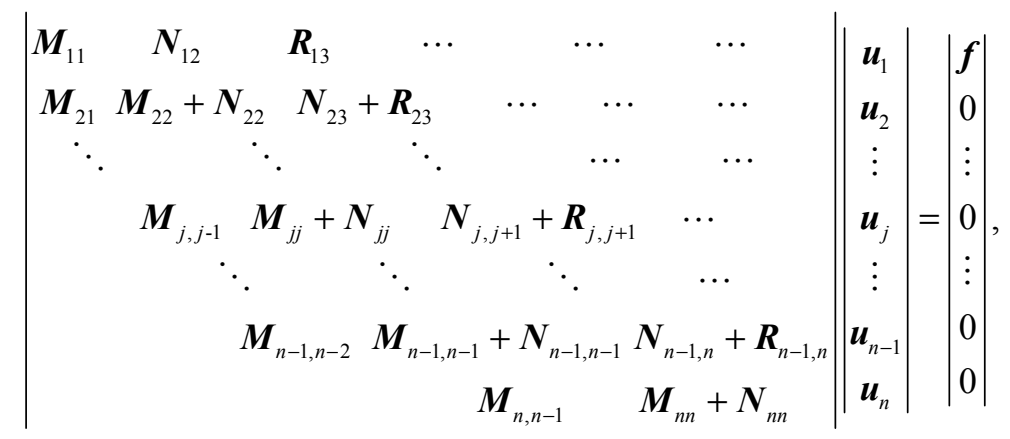

where $\boldsymbol{R}=\left(\boldsymbol{G} \boldsymbol{G}^{-1}\right) \boldsymbol{N}$.

The resulting new matrix can also be split into two parts, self-interaction matrices and extrapolation matrices. Equation (31) can be rewritten as 


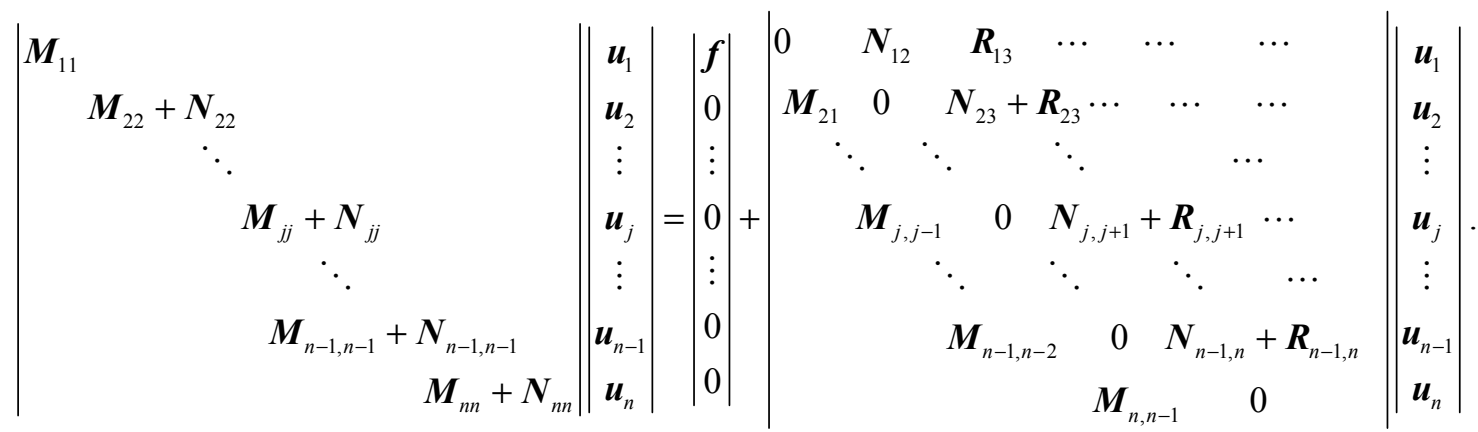

We compress our notation so that equation (32) becomes

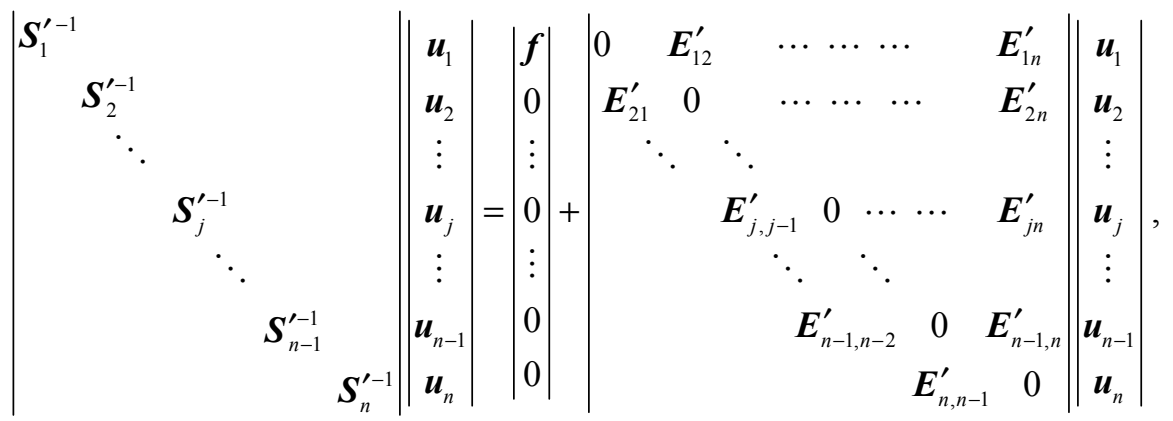

where $S_{i}^{\prime}$ and $\boldsymbol{E}_{i j}^{\prime}$ represent the sub-blocks related to the self-interaction operators and the extrapolation operators, respectively. With a number of iterations, the general formula is

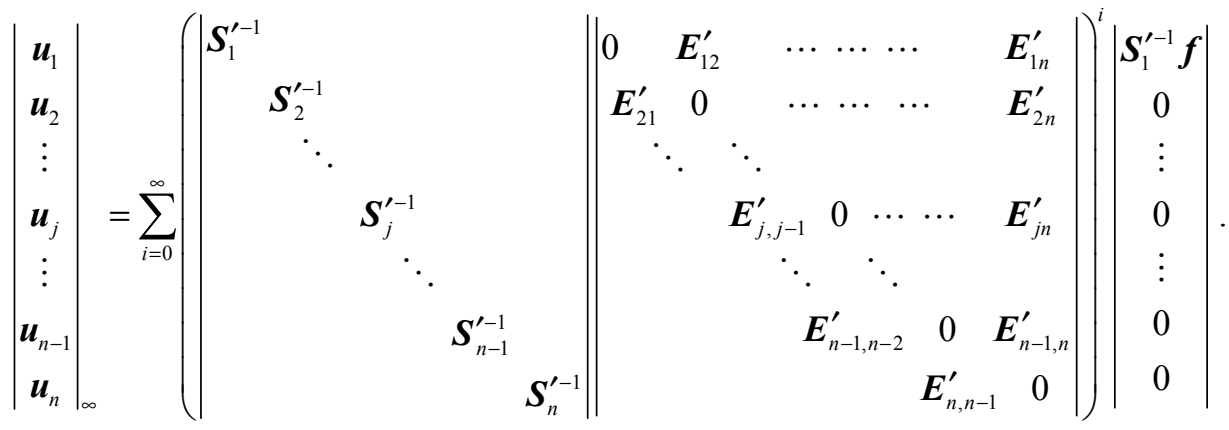

It seems that the form of equation (34) is similar to that of equation (20), but the self-interaction operators and extrapolation operators in equation (34) have had different physical meanings. The first scheme physically associates the self-interaction operator with each boundary itself, whereas the second relates the self-interaction operator to each subregion itself. The extrapolation operator is interpreted in the first scheme as cross-interactions between different boundaries in a subregion, but in the second scheme as cross-interactions between different subregions in a whole model. For the model shown in Figure 2, the Born series form of the second scheme can be written as

$$
\left|\begin{array}{l}
\boldsymbol{u}_{1} \\
\boldsymbol{u}_{2} \\
\boldsymbol{u}_{3}
\end{array}\right|_{\infty}=\sum_{i=0}^{\infty}\left(\left|\begin{array}{lll}
\mid \boldsymbol{S}_{1}^{\prime-1} & & \\
& \boldsymbol{S}_{2}^{\prime-1} & \\
& & \boldsymbol{S}_{3}^{\prime-1}
\end{array}\right| \begin{array}{ccc}
0 & \boldsymbol{E}_{12}^{\prime} & \boldsymbol{E}_{13}^{\prime} \\
\boldsymbol{E}_{21}^{\prime} & 0 & \boldsymbol{E}_{23}^{\prime} \\
0 & \boldsymbol{E}_{32}^{\prime} & 0
\end{array} \mid\right)^{i}\left|\begin{array}{c}
\boldsymbol{S}_{1}^{\prime-1} \boldsymbol{f} \\
0 \\
0
\end{array}\right| .
$$

\section{Numerical tests}

The two different BEM+Born series methods are tested to show their effectiveness by modeling a homogeneous layered model, compared with the full-waveform BE numerical solution. All computations for these examples are performed with frequency range $0-40 \mathrm{~Hz}$, with receivers along the surface and the source located at $(1500 \mathrm{~m}, 0 \mathrm{~m})$. The dimensions of the model are $3000 \mathrm{~m}$ horizontally and $1000 \mathrm{~m}$ vertically. Figure 2 shows a homogeneous layered model consisting of four subregions $\Omega_{i}(i=1,2, \ldots, 4)$ with the velocities $(\mathrm{km} / \mathrm{s})$ indicated in the figure. The source is a minimum-phase Gauss wavelet with a central frequency of $15 \mathrm{~Hz}$. A variable element dimension technique is adopted in the program implementation $(\mathrm{Fu}, 1996)$ to improve compu- 
tation speed because sampling at three elements per wavelength is sufficient to ensure the accuracy of the results (Campillo, 1987). Since the wavelength is a function of frequencies and velocities, the dimension of boundary elements for each subregion in a model is computed according to the medium velocity and the computational frequency. The model is then automatically discretized in terms of updated element dimensions for each computational frequency. During the numerical implementation, an absorbing boundary element technique ( $\mathrm{Fu}$ and $\mathrm{Wu}, 2000)$ is introduced to truncated edges of interfaces to handle artificial reflections arising at the edges of the domain of computation. Shown in Figures $3 \mathrm{a}$ and $3 \mathrm{~b}$ are the stacked sections calculated by the full-waveform BE numerical method and the finite-difference method made by Seismic Un*x (http://www.cwp.mines.edu/cwpcodes/), respectively. We see a rather good agreement between them, which indicates that the $\mathrm{BE}$ method and the implementing computation program are correct and valid.

The first-order iterative solution of the first scheme is $\boldsymbol{S}_{1}^{-1} \boldsymbol{f}$, which represents the reflection wave from the interfaces $\Gamma_{1}$ (Figure 4a). At the third iteration, the reflection (Figure 4b) from the interfaces $\Gamma_{2}$ appears because of the term $\boldsymbol{S}_{1}^{-1} \boldsymbol{E}_{12} \boldsymbol{S}_{2}^{-1} \boldsymbol{E}_{21} \boldsymbol{S}_{1}^{-1} \boldsymbol{f}$. At the fifth iteration, the reflections from all interfaces are shown in Figure $4 \mathrm{c}$ in good comparison to Figure 6 obtained by the full-waveform BE numerical solution. For the second method, the first-order and second-order Born approximations (Figures $5 \mathrm{a}$ and $5 \mathrm{~b}$ ) are similar to those of the first method. The convergence rate of the second method, however, becomes faster after the third iteration and the reflections from all interfaces are obtained at the fourth iteration (Figure 5c). The stacked sections in Figures 7 and 8 further verify the above analyses through the comparison with Figure $3 \mathrm{a}$ obtained by the full-waveform BE numerical solution. Both the numerical schemes avoid the inversion of the relatively much larger boundary integral equation matrix of a full-waveform BE method and hence save computing time and memory greatly, especially for the cases of high frequencies and multisource seismic survey.

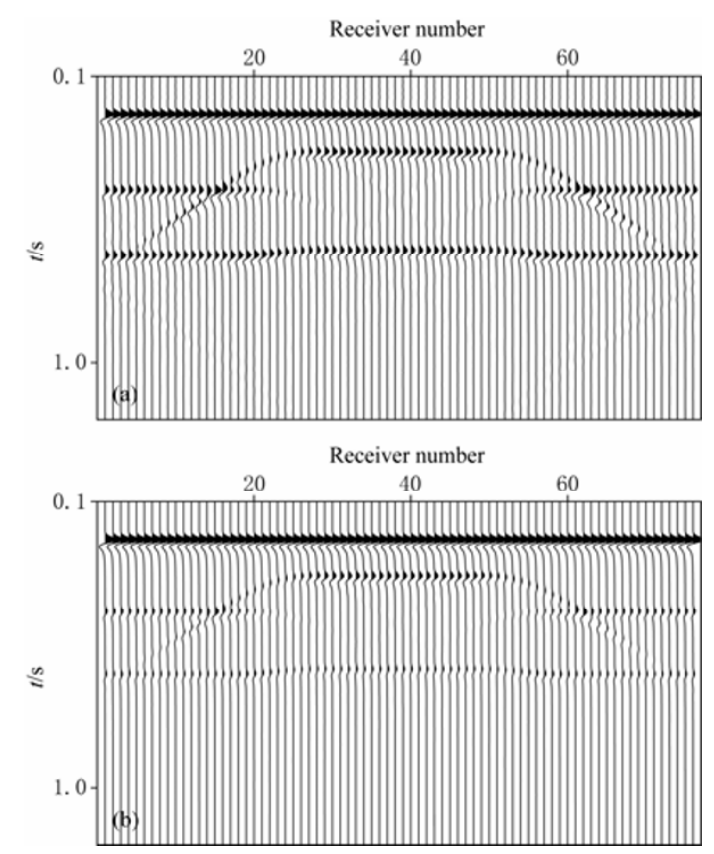

Figure 3 Seismic sections for a multilayered model by the full-waveform BE method (a) and the finite-difference method (b).
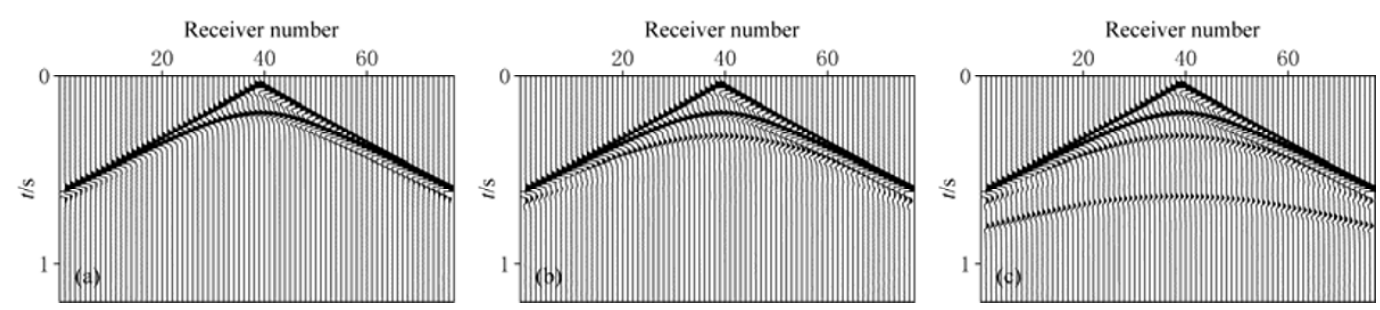

Figure 4 Synthetic seismograms for a multilayered model with the different-order Born approximations by the first scheme. (a) First-order Born approximation; (b) second-order Born approximation; (c) fourth-order Born approximation.

\section{Convergence analysis}

For the BEM+Born modeling schemes, $\boldsymbol{S}^{-1} \boldsymbol{E}$ is a linear bounded operator mapping. Take the model in Figure 1 for an example. The mapping $\boldsymbol{S}^{-1} \boldsymbol{E}$ of the first scheme is 

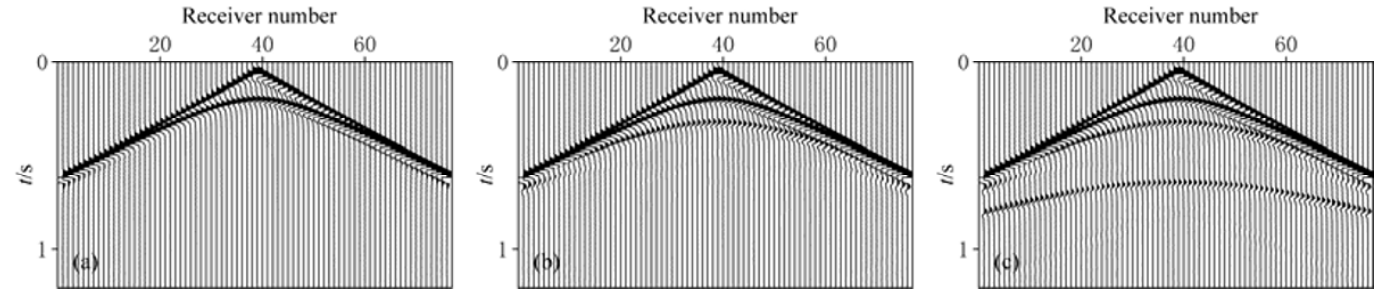

Figure 5 Synthetic seismograms for a multilayered model with the different-order Born approximations by the second scheme. (a) First-order Born approximation; (b) second-order Born approximation; (c) third-order Born approximation.

$$
\begin{aligned}
& \begin{array}{ll}
0 & \boldsymbol{S}_{1}^{-1} \boldsymbol{E}_{12}
\end{array} \\
& \begin{array}{lll}
\boldsymbol{S}_{2}^{-1} \boldsymbol{E}_{21} & 0 & \boldsymbol{S}_{2}^{-1} \boldsymbol{E}_{23}
\end{array} \\
& \ddots \quad \ddots \quad \ddots \\
& \boldsymbol{S}_{j}^{-1} \boldsymbol{E}_{j, j-1} \quad 0 \quad \boldsymbol{S}_{j}^{-1} \boldsymbol{E}_{j, j+1} \\
& \ddots \quad \ddots \quad \ddots \\
& \begin{array}{ccc}
\boldsymbol{S}_{n-1}^{-1} \boldsymbol{E}_{n-1, n-2} & 0 & \boldsymbol{S}_{n-1}^{-1} \boldsymbol{E}_{n-1, n} \\
\boldsymbol{S}_{n}^{-1} \boldsymbol{E}_{n, n-1} & 0
\end{array}
\end{aligned}
$$

For the second scheme, the mapping $\boldsymbol{S}^{-1} \boldsymbol{E}$ is

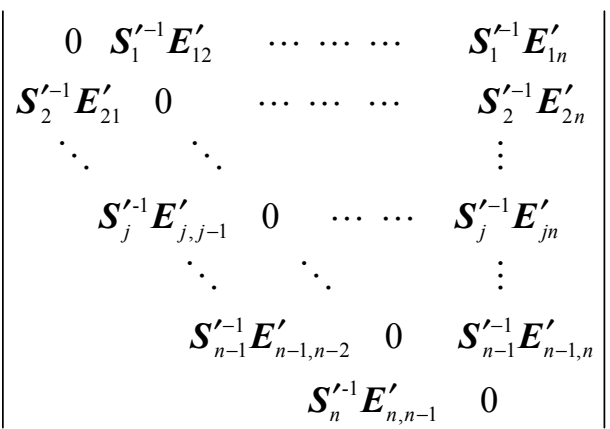

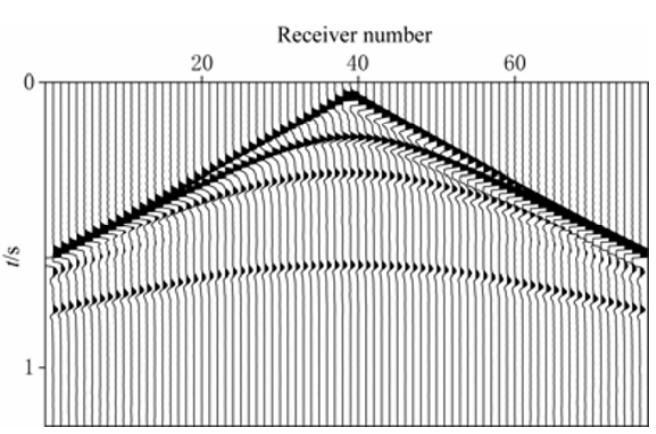

Figure 6 Synthetic seismogram for a multilayered model by the full-waveform BE method.

In comparison with the mapping $\boldsymbol{S}^{-1} \boldsymbol{E}$ of the first scheme, that of the second one has $(n-2)(n-1) / 2$ extra bounded operators because of elimination of the unknowns. And then we compare the reflections from the interface $\Gamma_{0}$ between the two schemes shown in Table 1. It can be found that the first-order and second-order Born approximations of the second scheme are similar to those of the first one, but at the fourth iteration the
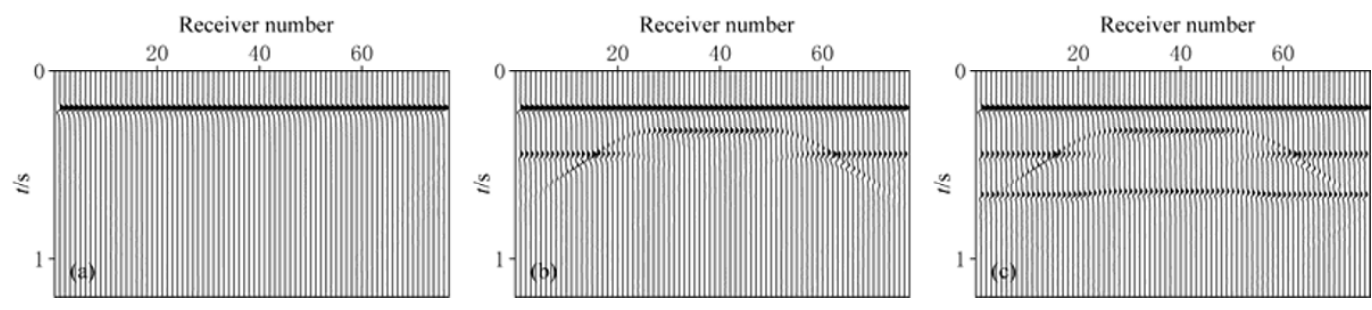

Figure 7 Seismic sections for a multilayered model with the different-order Born approximations by the first scheme. (a) First-order Born approximation; (b) second-order Born approximation; (c) fourth-order Born approximation.
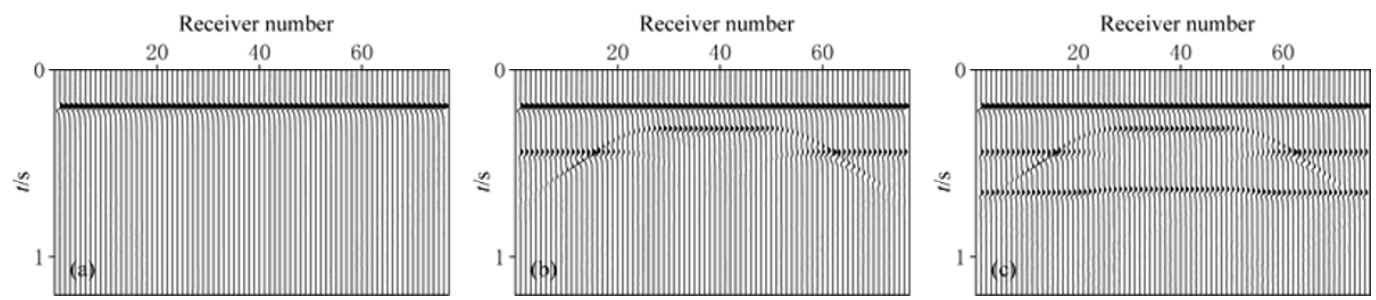

Figure 8 Seismic sections for a multilayered model with the different-order Born approximations by the second scheme. (a) First-order Born approximation; (b) second-order Born approximation; (c) third-order Born approximation. 
second scheme has an extra scattering term $R_{1321}$, which confirms theoretically the modeling results shown in Figure $5 \mathrm{c}$. After the fourth iteration, the second scheme has more extra scattering terms and obtains the diffracted /reflected waves from the next layer for each iteration. It can deduced from Table 1 that the first scheme requires $2 n-1$ iterations to obtain the diffracted /reflected waves from the $n$th layer, while the second scheme needs $n+1$ iterations only. In addition, the self-interaction operators of the second scheme account for not only the interaction of each boundary with itself as those of the first scheme, but also the cross-interaction between different boundaries in the same subdomain. The extrapolation operators of the second scheme include cross-interactions between these boundaries in different subdomains. Therefore, these differences in the mapping $\boldsymbol{S}^{-1} \boldsymbol{E}$ may be considered to accelerate the convergence rate of the second scheme.

Table 1 Comparison of the reflections observed at interface $\Gamma_{0}$ and the corresponding approximation terms from 1 st to 6 th order between the two schemes

\begin{tabular}{cll}
\hline \multirow{2}{*}{ Order of the approximate term } & \multicolumn{1}{c}{ Reflections from interfaces observed at interface $\Gamma_{0}$} \\
\cline { 2 - 3 } & \multicolumn{1}{c}{ Scheme 1 } & \multicolumn{1}{c}{ Scheme 2 } \\
\hline 0th & $R_{1}: \boldsymbol{S}_{1}^{-1} \boldsymbol{f}$ & $\boldsymbol{S}_{1}^{\prime-1} \boldsymbol{f}$ \\
$1 \mathrm{st}$ & 0 & 0 \\
$2 \mathrm{nd}$ & $R_{121}: \boldsymbol{S}_{1}^{-1} \boldsymbol{E}_{12} \boldsymbol{S}_{2}^{-1} \boldsymbol{E}_{21} \boldsymbol{S}_{1}^{-1} \boldsymbol{f}$ & $\boldsymbol{S}_{1}^{\prime-1} \boldsymbol{E}_{12}^{\prime} \boldsymbol{S}_{2}^{\prime-1} \boldsymbol{E}_{21}^{\prime} \boldsymbol{S}_{1}^{\prime-1} \boldsymbol{f}$ \\
$3 \mathrm{rd}$ & 0 & $R_{1321}: \boldsymbol{S}_{1}^{\prime-1} \boldsymbol{E}_{13}^{\prime} \boldsymbol{S}_{3}^{\prime-1} \boldsymbol{E}_{32}^{\prime} \boldsymbol{S}_{2}^{\prime-1} \boldsymbol{E}_{21}^{\prime} \boldsymbol{S}_{1}^{\prime-1} \boldsymbol{f}$ \\
4 th & $R_{12321}+R_{12121}$ & $R_{12321}+R_{12121}+R_{14321}$ \\
5 th & 0 & $R_{154321}+R_{121321}+R_{124321}+R_{134321}+R_{123231}+R_{132121}$ \\
6 th & $R_{1212121}+R_{1212321}+R_{1232121}+R_{1232321}+R_{1234321}$ & $R_{1212121}+R_{1212321}+R_{1232121}+R_{1232321}+R_{1234321}+\cdots+R_{1654321}$ \\
\hline
\end{tabular}

To analyze the relationship between matrix norm and convergence rate, we calculate the two norms of matrices $\boldsymbol{S}^{-1}$ and $\boldsymbol{E}$ at certain frequencies for the two schemes respectively. For the first scheme, the norms of matrices $S^{-1}$ are less than 1.0 and grow with the increasing of frequency, while the norms of matrices $\boldsymbol{E}$ decrease largely with the growing frequency. For the second scheme, the norms of matrices $\boldsymbol{S}^{-1}$ are also less than 1.0 but larger than those of the first scheme, while the norms of matrices $\boldsymbol{E}$ are smaller. As shown in Table 2 , the CPU time of the second scheme is about one half of that of the first scheme. By comparison we find that faster convergence may be usually achieved as the norms of $\boldsymbol{S}^{-1} \boldsymbol{E}$ approach zero. The CPU time of the full-waveform $\mathrm{BE}$ numerical method to solve the matrix equation at $25 \mathrm{~Hz}$ is $122.4 \mathrm{~s}$. In comparison, the two schemes significantly improve computational efficiency.

Table 2 The matrix norms and CPU time for the two modeling schemes at $25 \mathrm{~Hz}$

\begin{tabular}{lrrrc}
\hline & $\left\|\boldsymbol{S}^{-1}\right\|$ & \multicolumn{1}{c}{$\|\boldsymbol{E}\|$} & $\left\|\boldsymbol{S}^{-1} \boldsymbol{E}\right\|$ & CPU Time (122.4s) \\
\hline Scheme 1 & 0.745 & 212.58 & 45.55 & $30.34 \mathrm{~s}$ \\
Scheme 2 & 0.916 & 6.60 & 2.64 & $15.89 \mathrm{~s}$ \\
\hline
\end{tabular}

Note: * denotes the CPU time of the full-waveform BE numerical method.

\section{Discussion and conclusions}

This paper formulates two different hybrid $\mathrm{BEM}+\mathrm{Born}$ series modeling schemes for wave propaga- tion simulation in multilayered media. The two schemes reduce the whole system-matrix to the algorithm of the sub-matrix system by applying the full-waveform BE method to the self-interaction operator and a Born series to the "not-to-be-inverted" part, and require up to an order of magnitude fewer computations than a pure BE method. Both the numerical schemes are validated by calculating synthetic seismograms for a homogeneous layered model, compared with the full-waveform BE numerical solution.

This paper also discusses the convergence rate of the two modeling schemes. The numerical results indicate that faster convergence may be usually achieved as the norms of $\boldsymbol{S}^{-1} \boldsymbol{E}$ approach zero. Moreover, the second modeling scheme has a faster convergence in comparison with the first one. For a $n$-layer model, the first scheme requires $2 n-1$ iterations to obtain the diffracted /reflected waves from the nth layer, while the second scheme needs $n+1$ iterations only. The two schemes have the advantage of requiring the inversion of only self-interaction type matrices, and so save computational time and memory storage greatly. But the disadvantage of them is that the convergence rate depends on the model and is difficult to predict.

In summary, both the BEM+Born series modeling schemes significantly improve computational efficiency and can be extended to complex geological structures. The numerical schemes promise to be more efficient 
forward modeling tools than pure BE methods.

Acknowledgments The research is jointly supported by National Natural Science Foundation of China (No.40830423) and National Basic Research Program of China (973 Program, 2009CB219403).

\section{References}

Abramowitz M and Stegun I A (1968). Handbook of mathematical functions. Dover Publ. Inc, New York.

Bakamjian B (1992). Boundary integrals: An efficient method for modeling seismic wave propagation in 3-D. 62th Ann. Internat. Mtg. Soc. Expl. Geophys. New Orleans, USA, Expanded Abstracts, 1232-1 235.

Bouchon M (1993). A numerical simulation of the acoustic and elastic wavefields radiated by a source on a fluid-filled borehole embedded in a layered medium. Geophysics 58: 475-481.

Campillo M (1987). Modeling of SH-wave propagation in an irregularly layered medium: Application to seismic profiles near a dome. Geophys Prospect 35: 236-249.

Chen $\mathrm{G}$ and Zhou H (1994). Boundary element modeling of nondissipative and dissipative waves. Geophysics 59: 113-118.

Dong W J, Bouchon M and Toksöz M N (1995). Borehole seismic-source radiation in layered isotropic and anisotropic media: Boundary element modeling. Geophysics 60: 735-747.

Fu L Y (1996). 3-D boundary element seismic modeling in complex geology. 66th Ann. Internat. Mtg., Soc. Expl. Geophys. Denver, CO, USA, Expanded Abstracts, 1239-1 242.
Fu L Y (2002). Seismogram synthesis for piecewise heterogeneous media Geophys J Int 150: 800-808.

Fu L Y and Mu Y G (1994). Boundary element method for elastic wave forward modeling. Acta Geophysics Sinica 37: 521-529 (in Chinese with English abstract).

Fu L Y and Wu R S (2000). Infinite boundary element absorbing boundary for wave propagation simulations. Geophysics 65: 596-602.

Liu E, Dobson A, Pan D M and Yang D H (2008b). The matrix formulation of boundary integral modeling of elastic wave propagation in $2 \mathrm{D}$ multi-layered media with irregular interfaces. Journal of Computational Acoustics 16(3): 381-396.

Liu E, Zhang Z, Yue J and Dobson A (2008a). Boundary integral modeling of elastic wave propagation in multi-layered $2 \mathrm{D}$ media with irregular interfaces. Communications in Computational Physics 3(1): 52-62.

Pao Y H and Varatharajulu V (1976). Huygens' principle, radiation conditions and integral formulas for the scattering of elastic waves. J Acoust Soc Amer 59: 1361-1 370.

Schuster G T (1985a). A hybrid BIE+Born series modeling scheme: Generalized Born series. J Acoust Soc Amer 77: 865-879.

Schuster G T (1985b). Solution of the acoustic transmission problem by a perturbed Born series. J Acoust Soc Amer 77: 880-886.

Schuster G T and Smith L C (1985). Modeling scatterers embedded in plane-layered media by a hydrid Haskel-Thomson and boundary integral equation method. J Acoust Soc Amer 78: 1387-1 394.

Yu G X, Li D P, Fu L Y and Jiang T (2009). Numeric simulation of boundary element-volume element wave equation in complex geologic structure. Oil Geophysical Prospecting 44(1): 107-111 (in Chinese with English abstract). 